\title{
L'élu et l'ingénieur : aménagements civils littoraux et politique en Bretagne (vers 1890-vers 1930)
}

The Elected Representative and the Engineer. Civil Development of the Coast and Politics in Brittany

Laurent Le Gall

\section{(2) OpenEdition}

\section{Journals}

Édition électronique

URL : http://journals.openedition.org/abpo/2788

DOI : 10.4000/abpo.2788

ISBN : 978-2-7535-3513-8

ISSN : 2108-6443

\section{Éditeur}

Presses universitaires de Rennes

\section{Édition imprimée}

Date de publication : 30 juin 2014

Pagination : 151-167

ISBN : 978-2-7535-3511-4

ISSN : 0399-0826

\section{Référence électronique}

Laurent Le Gall, «L'élu et l'ingénieur : aménagements civils littoraux et politique en Bretagne (vers 1890-vers 1930) », Annales de Bretagne et des Pays de l'Ouest [En ligne], 121-2 | 2014, mis en ligne le 30 juin 2016, consulté le 01 mai 2019. URL : http://journals.openedition.org/abpo/2788 ; DOI : 10.4000/ abpo. 2788 


\title{
L'élu et l'ingénieur
}

\section{Aménagements civils littoraux et politique en Bretagne (vers 1890-vers 1930)}

\author{
Laurent LE GALL \\ Maître de conférences en histoire contemporaine, CRBC - \\ Université de Bretagne occidentale
}

Pour l'historien du politique qui aborde le rivage, les aménagements du littoral semblent, a priori, ne relever ni de sa sphère d'intervention ni de ses domaines de compétence. Ravalé chez la plupart de ses contemporains à l'étude de ce qui apparaît classiquement comme son terrain d'investigation - de l'élection à la vie parlementaire, pour faire bref -, condamné par certains à ne s'intéresser qu'à des objets proprement " politiques ", ce dernier ne serait-il donc pas en mesure d'apporter sa pierre de touche à une histoire globale du littoral? Il ne s'agit bien évidemment pas de trancher cette question mais de souligner que les aménagements civils côtiers ont pourtant bien de quoi nourrir sa réflexion. À la croisée des revendications individuelles et/ou collectives et des attentes - ou des résistances - de l'État, l'aménagement progressif d'un littoral organisé au service des activités humaines a été doté de nouveaux enjeux à la fin du XIXe siècle. Alors que le champ politique gagnait davantage en autonomie et se stratifiait, l'anthropisation " volontariste " des côtes mettait en jeu (et en œuvre) des prises de décision qui relevaient désormais autant d'une compétence et d'un primat techniques et étatiques que d'une implication des élus soucieux d'impulser et de contrôler des projets.

En faisant de l'élu et de l'ingénieur des Ponts et Chaussées les deux figures génériques qui commandèrent en partie aux destinées de l'aménagement portuaire, nous souhaitons nous interroger sur les interactions de la sphère administrative et de la sphère politique et sur leur concurrence et/ou leur complémentarité dans le processus décisionnel qui présida à cette organisation du littoral. Au cours d'une période - les années 18901930 - marquée par la spécialisation des tâches et la professionnalisation des agents du champ politique, comment des élus locaux devinrent-ils des bâtisseurs parce que les logiques du marché démocratique supposaient 
qu'ils soient de plus en plus à l'écoute des attentes de leur électorat? Des projets furent-ils au cœur d'une entreprise électorale ou ne dépassèrentils que très rarement le stade des simples décisions techniques engageant moins la collectivité dans son entier que ceux qui y étaient sectoriellement intéressés? Des réponses que nous essaierons d'apporter à ces questions en nous intéressant aux aménagements civils portuaires en Bretagne pourrait dépendre - c'est ce que nous espérons - une meilleure compréhension de ce que signifia la mise en place de l'ordre démocratique dans les villes et les villages du littoral.

\section{La cale et la jetée : les faux-semblants d'un aménagement vidé de sens politique}

\section{Aménager : une décision portée par deux logiques antagonistes?}

En apparence, la construction d'un môle ou d'une jetée dépendait uniquement d'une somme de décisions à la fois éminemment techniques et encadrées par toute une série de règles et de règlements qui mettaient aux prises des élus locaux - conseils municipaux, d'arrondissement et général -, l'administration préfectorale et l'institution des Ponts et Chaussées ${ }^{1}$. Cette impression était d'ailleurs d'autant plus fondée aux yeux des agents de l'État qu'elle reposait sur une double constatation : malgré la grande loi municipale du 5 avril 1884 qui avait conféré une personnalité juridique à la commune, la vie politique locale semblait être dénuée d'autres enjeux que ceux de la gestion de la collectivité ${ }^{2}$; le domaine de l'aménagement en général - de la voirie à la cale en passant par l'école - paraissait relever exclusivement de compétences spécifiques que ne possédaient qu'exceptionnellement des édiles dont l'État attendait avant tout qu'ils sachent engager des procédures puis les faire respecter. Dans une telle configuration, l'assemblée communale était réduite à son rôle de porte-parole d'un intérêt particularisé, à une époque où la division des tâches administratives et techniques mais aussi la valorisation de l'expertise dans quelque domaine que ce soit ${ }^{3}$ ne pouvaient que l'exclure de la plus grande part de la décision. Délibérer sur la demande d'un ouvrage portuaire puis voter

1. Pour le détail des opérations présidant à la construction des infrastructures portuaires, nous renvoyons à la thèse de doctorat en histoire contemporaine de Françoise Sioc'Han-Monnier, La construction et l'évolution des ports en Bretagne aux $19^{e}$ et $20^{e}$ siècles (Jean-Yves ANDRIEux [dir.]), Université de Haute-Bretagne, 1998. Du même auteur, «Projets de travaux maritimes, sûreté des ports et des rades, liberté du commerce et de la navigation (XIX-éébut XX ${ }^{\mathrm{e}}$ siècle) ", dans LE BouËDEc, Gérard et CHAPPÉ, François (dir.), Pouvoirs et littoraux du $X^{e}$ au $X X^{e}$ siècle. Actes du Colloque international de Lorient (24, 25, 26 septembre 1998), Rennes, PUR, 2000, p. 509-515.

2. FougĖRe, Louis, MACHELOn, Jean-Pierre et Monnier, François (dir.), Les communes et le pouvoir. Histoire politique des communes françaises de 1789 à nos jours, Paris, Presses Universitaires de France, 2002, p. 387-393.

3. Voir, par exemple, les réflexions que développe Éric PhÉLIPPEAU, L'invention de l'homme politique moderne. Mackau, l'Orne et la République, Paris, Éditions Belin, 2002, p. 222-236. 
une subvention à la toute fin de l'instruction du dossier constituaient les manifestations de son action et, par là même, les symptômes de son étroite marge de manœuvre. Demandé par des édiles, projeté par l'ingénieur ordinaire sur injonction du préfet, visé par l'ingénieur en chef puis transmis au ministre des Travaux publics pour examen par le conseil général des Ponts et Chaussées, l'objet de l'aménagement d'une portion du littoral était censé consacrer non seulement la solidité et l'effectivité d'une chaîne de décisions administratives mais aussi la maîtrise dont disposait l'État - une justification de sa légitimité - de faire coïncider l'intérêt général et les intérêts particuliers.

Cette question de l'intérêt général était au cœur même d'une idéologie des agents de l'État telle qu'elle s'exprimait généralement tout au long de la procédure. Réfutant le caractère exclusivement privé sur lequel l'ingénieur ordinaire avait bâti son argumentation pour rejeter la pétition de 80 marins demandant la création d'une cale de 20 mètres de long susceptible de leur faciliter l'accostage à l'entrée du Bélon, le député de Kerjégu écrivit au préfet le 5 mai 1897 :

" J'ajouterai qu'il n'est pas exact que la cale à construire ne réponde qu'à un intérêt privé. Elle donnerait, en effet, satisfaction à un ensemble considérable d'intérêts privés, ceux de tous les pêcheurs de la région qui viennent apporter sur ce point les produits de leur industrie et y trouvent leur principal débouché. Or, vous le savez, Monsieur le Préfet, c'est précisément la réunion, la collectivité des intérêts privés qui, par leur groupement, arrivent à former l'intérêt public. Je me crois donc fondé à soutenir qu'il y a bien, dans la circonstance, un intérêt public en jeu ${ }^{4}$."

En réponse aux sollicitations des populations littorales et du conseil municipal de Plozévet qui espérait bénéficier des crédits affectés exceptionnellement par le gouvernement en 1919 pour la transformation des petits ports, l'ingénieur ordinaire se retrancha, dans sa réponse du 25 mai 1920, derrière un argument utilisé à maintes reprises : estimant que «L'amélioration du port de Pors-Poulhan n'intéress[ait] guère que les pêcheurs de la localité au nombre de 130 environ, armant une trentaine de canots, et, par suite, ne présent[ait] qu'un intérêt tout à fait local ${ }^{5}$ ", il invita son administration à repousser la demande du brise-lames. Ainsi, la définition du bien commun dont les agents en charge des dossiers avaient compris qu'elle pèserait lourdement au cours de la négociation - d'où la multiplication des propos vétilleux de part et d'autre - commanda fréquemment le positionnement de ces derniers à chaque étape de la procédure.

Faire que le succès d'un projet repose sur un quitus purement administratif, c'était rejeter par là même le principe d'une démocratie de la participation à la décision. C'était fonder aussi une étanchéité entre, d'une part, un champ politique traversé d'enjeux de pouvoir et de contradictions et, d'autre part, les domaines d'intervention d'un État avide de défendre son

4. Arch. dép. du Finistère, 4 S 69.

5. Arch. dép. du Finistère, 4 S 1426. 
primat et ses prérogatives. Soumis au bon vouloir de techniciens qui disposaient de deux atouts par rapport aux élus - une connaissance des dossiers fondée sur une expertise à prétention scientifique et une aptitude à incarner la supériorité du bien commun ${ }^{6}$-, les édiles ne pouvaient que constater l'infériorité de leur rôle au cours d'une démarche qui se révélait souvent longue, sinueuse et susceptible de ne pas aboutir. Du point de vue de ces derniers, il ne faisait alors aucun doute que la prééminence des ingénieurs dans la conduite et la réussite des opérations consacrait cette mise à l'écart que l'État leur imposait et contre laquelle rien n'y ferait (puisque ni les pétitions ni les récriminations ni les requêtes ne semblaient infléchir le pouvoir de contraindre dont disposaient lesdits ingénieurs). Alimentée par de très nombreux heurts entre la municipalité et l'administration des Ponts et Chaussées, la chronique de l'amélioration du port de Roscoff qui se conclut par la création d'un second bassin - projeté en 1907, il fut mis en service en 1930-1931 - traduisit, au-delà des incompréhensions réciproques, le conflit de deux institutions bataillant pour affirmer leur propre légitimité et la victoire de la seconde sur la première ${ }^{7}$. Quant à l'intervention du député du Finistère et conseiller général de Fouesnant, Louis Hémon, lors de la séance du conseil général du 22 août 1890, elle sanctionna in fine l'échec d'une demande émanant d'un des organes de la représentation populaire :

" Je regrette que l'Administration ait refusé l'établissement d'un quai à Lanriec; il y a là des intérêts considérables qui réclament une satisfaction; M. l'Ingénieur en chef du service maritime trouve la dépense exagérée; je demande au Conseil de renouveler son vœu en faveur de l'établissement de ce quai ${ }^{8}$."

L'État considéré comme cette superstructure lointaine et toute-puissante semblait incapable de promouvoir les formes d'une concertation locale à laquelle des élus prétendaient prendre part au nom de l'onction démocratique que leur conférait le suffrage universel. Réduits à n'être que des intermédiaires dans des affaires qui les concernaient au premier chef, il leur restait alors, et en dernier recours, à s'abriter derrière un discours rôdé de longue date dont le registre varia inlassablement entre l'amertume et l'incompréhension ${ }^{9}$.

6. Ils étaient d'autant plus à même d'épouser puis de véhiculer l'idéologie d'un État au service du bien commun que leur formation intellectuelle et leur statut de fonctionnaire les conduisaient à en être persuadés. Voir, à ce propos, les analyses de Pierre RosAnVALLon, L'État en France de 1789 à nos jours, Paris, Éditions du Seuil, coll. " Points histoire ", 1993 [1990], p. 221-225.

7. HÉRUBEL, Marcel, Le port de Roscoff. Étude d'économie maritime, Paris, Société d'éditions géographiques, maritimes et coloniales, 1924, p. 48-49; САВIOCH, Marie-Bernadette, Les ports de Roscoff, Sieck et Moguériec de 1850 à 1914, mémoire de maîtrise (Claude GeSLIN [dir.]), Université de Bretagne Occidentale, 1993, p. 142-185.

8. Arch. dép. du Finistère, 1 N 100.

9. Exemplaire des arguments utilisés par les élus en proie aux rebuffades de l'Administration, l'intervention de Georges Le Bail, conseiller général du canton de PlogastelSaint-Germain, lors de la séance du conseil général du 2 octobre 1924, insista sur l'unité du discours édilitaire pour essayer de disqualifier la position des représentants de l'État : 
Cette vision simplificatrice mérite toutefois d'être amendée à condition de replacer tout projet d'aménagement du littoral dans une économie de la décision bien plus complexe que le bel ordonnancement des dossiers - des délibérations du conseil municipal à l'accord ministériel en passant par la tracé des plans - ne le laisse supposer au prime abord. Répondant à la normalisation du discours administratif, le lissage de la proposition, telle qu'elle était soumise par l'ingénieur à son autorité de tutelle, correspondait moins au souhait de dresser la généalogie d'un consensus - elle aurait supposé que tous les points de vue soient représentés - qu'à la nécessité d'une mise en forme administrative dictée par les besoins de la synthèse. La verticalité de la décision finale ne saurait toutefois masquer les tractations et les rétractations multiples, les nombreuses interactions entre des agents qui n'étaient pas réduits à ce que leur rôle et leurs compétences respectives les condamnaient en théorie à être. À terme, la formalisation d'un projet selon un modèle déterminé de longue date - le rapport final des ingénieurs adressé au ministre des Travaux publics en était la quintessence - recouvrait, en effet, un fractionnement d'épisodes qui avaient engagé différents interlocuteurs et une somme de micro-décisions ${ }^{10}$. Passés sous silence dans les rapports définitifs, les échanges entre les parties impliquées dans l'élaboration des aménagements portuaires étaient pourtant on ne peut plus fréquents. Ils prirent des tournures différentes. L'ingénieur se rendant sur les lieux et cherchant à composer avec le conseil municipal ou les pétitionnaires fut un classique du genre (à Moëlan-sur-mer en $1897^{11}$, LampaulPlouarzel en $1925^{12}$, Erquy en $1933^{13}$ ). Le conseiller général ou le député

« J'insiste avec mon Collègue Le Coz [conseiller général du canton de Pont-l'Abbé] sur la gravité de la situation faite à Penmarc'h par la lutte des flots. Dans cette commune, par exemple, on est contraint de réserver tous les fonds communaux pour la réparation des dommages causés par la mer, si bien que les travaux d'amélioration du port ne peuvent être entrepris. Il est regrettable que l'État persévère dans sa manière d'opérer et qu'une confusion existe entre les simples travaux de défense du littoral et les autres " (Arch. dép. Finistère, 1 N 141).

10. Concernant la genèse de la décision administrative, on lira avec profit l'ouvrage de Florian Charvolin, L'invention de l'environnement en France. Chroniques anthropologiques d'une institutionnalisation, Paris, Éditions La Découverte, 2003. L'auteur note : «En s'attachant à restaurer la part de vivacité de l'archive administrative et en se penchant sur la littérature grise, on a pu envisager en détail les raisons pour lesquelles d'une part des opérations pensées largement comme analytiquement posées par l'observateur étaient en fait des trouvailles pratiques permettant à chaque protagoniste de s'aligner avec les autres sans entrer dans le détail, et, d'autre part des opérations pensées largement comme abstraites et formelles qui n'en étaient pas moins des opérations réalisées localement ", p. 107-108.

11. Arch. dép. du Finistère, 4 S 69. L'ingénieur ordinaire nota dans son rapport du 31 mai 1897 : «Deux convocations officieuses des pétitionnaires et deux visites des lieux ont été faites tout récemment. Peu de marins ont répondu à l'appel (six la première fois et cinq la deuxième), mais les renseignements recueillis permettent de penser que l'affaire se présente dans des circonstances analogues à celles de 1894. "

12. Arch. dép. du Finistère, 4 S 1372, rapport du subdivisionnaire du 4 novembre 1925.

13. Sioc'HAN-Monnier, Françoise, La construction et l'évolution des ports en Bretagne aux $19^{e}$ et $20^{e}$ siècles, op. cit., p. 76-77. 
usant de son influence pour faire accepter le projet du conseil municipal en fut un autre : en 1910, le syndic des marins de Plogoff pria le député radical Georges le Bail d'intercéder auprès de l'Administration en vue de l'établissement rapide d'un plan incliné et d'une plateforme avec treuil de hissage ${ }^{14}$. Quant à la consultation officieuse de l'ingénieur des Ponts et Chaussées par le maire, sa mention exceptionnelle dans les sources - elle est évoquée dans le registre des délibérations du conseil municipal de Camaret en $1922^{15}$ - signala davantage une entorse à la règle implicite de la très stricte répartition des rôles au cours de la fabrication des dossiers qu'une réelle absence de contact entre des agents qui se seraient ignorés ${ }^{16}$. Dans ce ménage à plusieurs partenaires (les interventions du maire et de l'ingénieur ne sauraient dissimuler celles, plus discrètes, du préfet, du conseiller d'arrondissement ou d'un parlementaire), l'engagement des uns dépendait des intentions des autres, ce qui obligeait chaque partie à une redéfinition du projet et à son actualisation au gré des discussions et des réunions préparatoires. À condition d'être attentif aux quelques indices qui attestent la porosité des camps, surtout au moment où se concrétisaient les propositions, l'on peut donc postuler, à la suite des travaux de Pierre Grémion sur le pouvoir périphérique, que l'autonomie des sphères administrative et politique fut contredite in situ par des échanges interpersonnels et des solidarités informelles. En générant une interpénétration de ces deux sphères, celle des élus et celle des fonctionnaires, la construction d'un quai ou d'une jetée participa incidemment à l'élaboration de ce " système politico-administratif local ${ }^{17}$ " fondé sur la nébulosité des rôles, l'ambivalence des fonctions, les transferts d'informations, les accointances diverses et, en fin de compte, la quête de la conciliation des points de vue ${ }^{18}$.

\section{La démocratie locale à l'épreuve de la logique étatique?}

Le caractère transactionnel de l'aménagement portuaire - car c'est bien d'une transaction qu'il s'agissait -, quelle qu'était la puissance respective des interlocuteurs en présence (le projet d'une cale dans une petite commune littorale ne pouvait pas prétendre susciter le même intérêt que la construction d'un bassin à flot dans un port réputé pour les forts volumes de son trafic), fut d'autant plus marqué que, depuis l'élection des conseils municipaux au suffrage universel et le renforcement des prérogatives des

14. Arch. dép. du Finistère, 4 S 1412, rapport du subdivisionnaire du 13 avril 1913.

15. Arch. dép. du Finistère, 4 S 172, séance du conseil municipal du 25 juin 1922.

16. Cabantous, Alain, Lespagnol, André et PÉRON, Françoise (dir.), Les Français, la terre et la mer, XIII -XXe siècle, Paris, Éditions Fayard, 2005, p. 538.

17. Pour reprendre le titre de la deuxième partie de l'ouvrage de Pierre GRÉMION, Le pouvoir périphérique. Bureaucrates et notables dans le système politique français, Paris, Éditions du Seuil, 1976, p. 151.

18. En guise de prolongement, on lira avec profit l'article de Jean-Michel EYMERI, "Frontières ou marches? De la contribution de la haute administration à la production du politique ", dans LagroYe, Jacques (dir.), La politisation, Paris, Éditions Belin, 2003, p. 47-77. 
assemblées locales sous la Troisième République, les élus étaient à la fois les administrateurs d'une collectivité d'habitants et les représentants d'une communauté de citoyens. À la fois maillons de cet ordre démocratique qui s'était imposé au fur et à mesure que ses tenants lui avaient donné corps et gestionnaires des intérêts particuliers d'un rassemblement d'individus, ces derniers pouvaient prétendre pallier leur infériorisation administrative par le fait qu'ils étaient les mandataires du peuple - des mandataires souvent fort bien élus puisque la multiplication des listes, au moment des scrutins, ne se soldait que rarement par un aussi grand émiettement des voix. Arguer de la délégation du peuple pour justifier une demande en vue de la construction ou de la réfection d'un ouvrage n'était bien évidemment pas chose nouvelle. Avant la consécration du suffrage en tant que mode d'exercice légitime de la souveraineté, les représentants des collectivités se prévalaient déjà de leur rôle de porte-parole pour essayer de faire admettre leurs projets. Reste que le consentement démocratique lui conféra une autre valeur, ce que les élus se privèrent rarement de rappeler. La question des pétitions corrobore d'ailleurs cette évolution. Nombreuses furent les entreprises qui émergèrent suite à la présentation d'une ou de plusieurs suppliques. Dans sa délibération du $1^{\mathrm{er}}$ décembre 1889, le conseil municipal de Locquirec " appuya[i]t " - et, pourrait-on ajouter, s'appuyait sur " une pétition des marins de la localité ${ }^{19}$ " pour faire valider sa requête, la construction d'un éperon capable d'abriter le mouillage fréquenté par cinq bateaux de pêche et un borneur. Prétendant parler " au nom des habitants du quartier de Kernevel ${ }^{20}$ ", deux élus de Larmor-Plage adressèrent pour la seconde fois, le 9 décembre 1925, une lettre à leur municipalité dans l'espoir d'obtenir le prolongement de la cale qu'utilisaient thoniers, langoustiers, sardiniers et bateaux de plaisance. Avec l'affermissement d'un ordre politique qui puisait sa force dans sa prétention à s'incarner par la voix de citoyens égaux et rationnels, les requêtes et les doléances écrites qui participaient de longue date à cette formulation des recours auprès des autorités changèrent de signification. Si le but des pétitions restait le même - alerter sur un besoin jugé impérieux -, leur intégration dans le système démocratique les requalifia immanquablement ${ }^{21}$. Ainsi, qu'elles aient été provoquées par quelques individus pour devenir ces palliatifs à une action municipale jugée par trop lente voire inexistante ou qu'elles aient été encouragées en sous-main par des élus conscients qu'il existait là un moyen de faire naître un projet, ces interpellations concrétisèrent des aspirations sur lesquelles rejaillissait désormais le cautionnement de la volonté populaire. Les espoirs mis dans la construction d'une cale, d'un môle ou d'un brise-lames matérialisaient autant une somme d'attentes par-

19. Arch. dép. du Finistère, 4 S 1350, rapport de l'ingénieur ordinaire du $1^{\text {er }}$ mars 1890.

20. Arch. dép. du Morbihan, 4 S 694.

21. Contamin, Jean-Gabriel, Contribution à une sociologie des usages pluriels des formes de mobilisation. L'exemple de la pétition en France, thèse de doctorat en science politique (Michel OfFERLÉ [dir.]), Université Paris 1, 2001. 
ticulières qu'une expression d'un marché démocratique irréductible à la seule procédure électorale.

Dans cette économie de la décision fortement concurrentielle - est-il besoin de le souligner -, à la croisée de la compétence administrative et des attentes exprimées par des assemblées délibératives au nom du bien-être de la communauté ou d'une de ses fractions, les élus entendirent disposer de l'atout démocratique pour essayer d'imposer leur vue. L'utilisation de cet atout, qui se mua fréquemment sous la plume des édiles en un argument d'autorité, put prendre plusieurs formes dont nous ne retiendrons que quelques-unes parmi les plus saillantes. En premier lieu, l'opposition d'une mémoire municipale de l'aspiration démocratique à une mémoire administrative de l'expertise technique fut exemplaire de l'enjeu que représenta pour le personnel politique son aptitude à s'abriter derrière cette aspiration. L'abandon d'un projet pour des raisons diverses - rapport négatif de l'ingénieur en chef sur recommandation de l'ingénieur ordinaire, budget communal incapable de supporter une partie des travaux - ne se traduisit qu'exceptionnellement par son enterrement. Les premières déconvenues ne grevant pas l'espoir de le faire aboutir un jour, l'ouvrage initialement demandé revenait sur la table du conseil municipal à intervalles réguliers. Des projets mirent des années et quelquefois des décennies avant de voir le jour. À propos du port du Conquet, l'ingénieur ordinaire rappela en 1880 que les premières études remontaient à 1771 et qu'elles avaient "été provoquées par une pétition des habitants adressée à l'intendant de Bretagne ${ }^{22}$ " . Aussi, pour obvier, dans cette course d'obstacles, à l'argumentaire de l'administration des Ponts et Chaussées qui, par l'intermédiaire des rapports successifs de son personnel, indexait la légitimité de son refus sur la légitimité de ses refus antérieurs, la parade que trouvèrent certaines assemblées communales reposa sur le même principe : celui de l'exhaussement d'une mémoire qui valorisait, elle, le caractère foncièrement démocratique de la demande projetée. Soucieuse de rivaliser avec une mémoire du déni élaborée et justifiée rapport après rapport, les dépositaires de la mémoire municipale se faisaient fort d'exprimer, quand bien même l'écriture de cette contre-mémoire était souvent fort maladroite et un tantinet réduite à quelques répétitions de principe, ce qui, d'après eux, devrait tôt ou tard faire pencher la balance en faveur de la proposition soumise au bon vouloir de l'Administration : la compétence démocratique et la reconnaissance qui en résultait. Délibérant, le 9 juillet 1934, sur le prolongement du môle de Lauberlach, un des petits ports de la presqu'île maraîchère de PlougastelDaoulas, le conseil municipal décida d'appuyer sa requête en énumérant les réclamations des riverains et les vœux de l'assemblée communale qui, depuis le début du siècle, avaient rythmé les projets d'aménagement de

22. Arch. dép. du Finistère, 4 S 1374. Se reporter aussi à l'article de Louis CHAURIS, "L'aménagement portuaire du Conquet (Finistère) : cent ans de discussions sur des projets (1771-1873) ", Bulletin de la Société Archéologique du Finistère, tome cxxvII, 1998, p. $399-415$. 
cette portion du littoral : étudié en 1910, repris en 1920 puis en 1926 « à la suite d'une pétition du syndicat des Inscrits maritimes ${ }^{23}$ ", le projet d'une cale facilitant le débarquement des produits de la pêche refaisait ainsi surface à la faveur d'une intensification du trafic maritime avec la ville de Brest.

La voix du peuple put servir, en second lieu, à justifier l'éclatement d'un contentieux. Quand une fin de non-recevoir du ministère des Travaux publics contrariait une demande qui avait été généralement faite à plusieurs reprises, la confrontation des points de vue pouvait déboucher sur une crise telle qu'elle mettait en péril la logique de conciliation - ce que nous avons essayé de montrer précédemment - qui prévalait habituellement. Objet de transactions à l'intérieur d'un cadre décisionnel qui était suffisamment plastique pour faciliter échanges, tractations et négociations, l'ouvrage projeté devenait alors cet objet de la discorde qui imposait que chaque camp fourbisse ses armes en fonction des prérogatives dont il était revêtu. Dans un contexte de crispation où un conseil municipal devait affronter un pouvoir administratif auréolé de sa toute-puissance bureaucratique $^{24}$, une des manières de peser - pour ne pas dire la seule - supposait de la part des élus qu'ils excipent de leur légitimité démocratique. Alors que la crise sardinière sévissait, le bras de fer qui opposa la municipalité concarnoise à l'Administration se solda ainsi par un coup d'éclat. L'ajournement d'un neuvième projet d'agrandissement du port - il prévoyait l'établissement de môles en avant du bassin d'échouage, entre la "ville close " et la jetée qui marquait l'entrée du port -, soumis au feu des critiques des ingénieurs et de certains représentants des pêcheurs, convainquit le maire, Billette de Villeroche et l'assemblée qu'il présidait, de démissionner. En se démettant de leur mandat le 31 décembre 1910, les élus misèrent sur leur va-tout démocratique pour imposer définitivement leur dessein. Un mois et demi après, les socialistes s'emparèrent de la mairie devenue vacante et l'on peut lire dans leur profession de foi :

"Question du port - Creuser le port afin de permettre, à toute marée, l'accostage à quai des bateaux sardiniers; Remettre en chantier le projet étudié par la municipalité précédente; l'étudier et le compléter s'il est nécessaire; en un mot donner sans retard à notre port la situation commerciale qu'il est en droit d'attendre ${ }^{25}$."

En invalidant momentanément les pratiques habituelles de la conciliation, toute crise permettait de rompre avec l'interpénétration des sphères administrative et politique. Pour autant, si la dynamique de l'intransigeance fondée sur l'opposition de la logique bureaucratique et de la logique démocratique grippait ponctuellement le processus décisionnel, elle n'entamait

23. Arch. dép. du Finistère, 4 S 1380.

24. Weber, Max, Économie et société, tome $1:$ Les catégories de la sociologie, Paris, Éditions Pocket, coll. "Agora ", 1995 [1971], p. 294-301.

25. Arch. dép. du Finistère, 3 M 480. Voir aussi le mémoire de maîtrise de Régis LE Floc'H, Concarneau de 1852 à 1914. Aspects économiques et sociaux (Claude GesLin [dir.]), Université de Bretagne Occidentale, 1993, p. 185-190. 
en rien l'existence du système politico-administratif local qui l'avait généré. En obligeant chaque camp à se retrancher derrière ses attributions, en confortant chaque agent dans son rôle, la situation de conflit sanctionnait moins la défaite d'un des partenaires que la pertinence d'un système suffisamment puissant pour rendre la crise passagère.

\section{L'aménagement portuaire : un enjeu communal ou un enjeu municipal?}

\section{Un objet digne d'un consensus politique}

La cale comme métaphore de l'aménagement civil portuaire participa à la constitution du système politico-administratif local. De sa transformation en un instrument possible du clivage politique qu'institua la mise en place de l'ordre démocratique, il n'y avait qu'un pas que l'analyse des sources invite cependant à ne pas franchir. Alors que l'aménagement portuaire consacra le positionnement des élus sur la base de leur compétence démocratique, l'on notera qu'il ne devint que très rarement un enjeu idéologique. Dans les villes où l'autonomisation du champ politique local et sa division ne cessèrent de gagner en intensité au fur et à mesure que l'organisation des formations partisanes permit à des individus de se solidariser avec une idéologie (le républicanisme, le socialisme, une des formes du conservatisme), l'annexion d'un projet par un camp fut exceptionnelle. Si, avec l'élaboration et la médiatisation des professions de foi, l'amélioration de la vie quotidienne et du cadre de cette vie devint la préoccupation principale des prétendants aux différentes charges électives, force est de constater que l'aménagement du littoral figura sur les supports de cette publicité électorale au même titre que les questions touchant à la voirie ou à la construction d'un bâtiment scolaire. Sur l'affiche de la liste républicaine d'union de 1919 qui égrena les mesures à prendre impérativement à Penmarc'h et se conclut par un retentissant " Nous ferons tout pour que la Mairie soit réellement la maison de tous, de l'union, de la concorde et du travail pour notre petite patrie ", les électeurs pouvaient lire aussi :

"En vue de l'amélioration de nos ports, nous ferons des démarches incessantes auprès de l'Administration supérieure et rappellerons à nos représentants à la Chambre leurs promesses ${ }^{26}$."

La même année, au Guilvinec, le tract de l'union républicaine fit valoir le même argument :

"Le port du Guilvinec prend chaque jour de l'importance. Au moment où il est question d'appliquer des crédits importants à l'amélioration des ports de pêche, vous avez intérêt à avoir à la tête de la commune une Municipalité sérieuse, capable de faire valoir vos besoins ${ }^{27}$." 
Moins qu'un argument de circonstance à l'usage des populations maritimes, la question de l'aménagement du littoral demeura un élément suffisamment déterminant dans presque tous les programmes électoraux pour ne pas être amalgamée à une seule et même famille politique. "Marins-Pêcheurs " d'Audierne, interpella en 1908 la liste communale d'entente républicaine : " Nous apporterons tous nos efforts à faire adopter dans le plus bref délai un projet d'amélioration du port ${ }^{28}$. " Près de trente ans après, dans cette circonscription du Cap-Sizun, le ton du placard du Parti communiste fut beaucoup plus offensif; pour autant, ce dernier n'omit pas de signaler qu'il restait attentif, au-delà de l'appel classique à l'union prolétarienne des « travailleurs de la mer et de l'usine ", aux problèmes du moment :

"Pour les marins, amélioration du port par la construction d'une écluse ainsi que le dragage de notre malheureux port, qui s'encombre de plus en plus, sous l'œil bienveillant de notre municipalité29."

Partie intégrante d'un projet politique municipal sur lequel s'arcboutèrent les candidats afin d'apparaître comme les garants les plus sûrs des intérêts de la commune et de sa prospérité, les ouvrages portuaires - à condition qu'ils aient une certaine ampleur - ne se parèrent pas d'enjeux idéologiques. Pour au moins trois raisons. À une époque où l'affermissement de l'ordre démocratique se nourrissait de la multiplication de l'offre politique et d'une différenciation croissante des opinions, tout projet délié d'un caractère hypothétiquement diviseur se trouvait aisément ravalé à ce qu'il avait de plus prosaïque et de plus contingent. Ainsi en allait-il de la construction d'une cale ou d'un môle quand le logement de l'instituteur était susceptible de déclencher un conflit résonnant de l'écho des batailles scolaires engagées à un niveau national. À la différence des affrontements concernant la place de l'Église dans la société française ou le choix d'une des alternatives révolutionnaires, l'ouvrage portuaire ne risquait pas de cristalliser clivages et tensions. L'argumentaire électoral que déploya Georges Le Bail et les réalisations qu'il entreprit au cours de sa vie édilitaire et parlementaire furent exemplaires de la non-politisation volontaire de l'aménagement du littoral. Héritier d'une dynastie républicaine qui s'était implantée dans la commune de Plozévet au début de la monarchie de Juillet, celui qui incarna, dans la Cornouaille occidentale du premier tiers du $\mathrm{xx}^{\mathrm{e}}$ siècle, un cacique radical doublé d'un entrepreneur politique $^{30}$, ne fit pas de l'amélioration des conditions d'accès au rivage un tremplin dans sa stratégie de conquête puis de conservation du pouvoir. Maire d'une commune maritime du canton de Plogastel-Saint-Germain, Le Bail n'en cautionna pas moins de son autorité l'aménagement de l'anse de Pors Poulhan ${ }^{31}$. Reste qu'il ne chercha guère à bâtir sa popularité et

28. Arch. dép. Finistère, 3 M 437.

29. Arch. dép. Finistère, 3 M 437.

30. BuRguiÈre, André, Bretons de Plozévet, Paris, Flammarion, coll. "Champs ", 1977 [1975], p. 216-231.

31. Arch. dép. Finistère, 4 S 248 et 4 S 1426. 
son bastion politique en investissant les ouvrages portuaires d'une charge polémique alors même qu'il eut à cœur d'endosser le rôle de porte-parole des populations de la frange littorale - il fut, par exemple, président de la commission de la Marine marchande à la Chambre des députés. Conscient qu'une des règles du marché démocratique supposait que la fructification d'un capital électoral dépendît de l'adéquation d'un message à un groupe social $^{32}$, Le Bail refusa de s'emparer des chantiers côtiers pour alimenter artificiellement la conflictualité politique et préféra rassembler les électeurs en utilisant le levier traditionnel de l'anticléricalisme du milieu maritime ${ }^{33}$. Aussi, les campagnes qu'il mena contre ses adversaires à l'échelle de sa circonscription ne laissèrent-elles qu'une place modeste à des considérations d'ordre matériel. La victoire à la députation en 1906, telle qu'il la relata dans un livre paru deux ans après, résonna toujours des combats acharnés que lui et ses partisans avaient menés contre une Église catholique soupçonnée de vouloir confisquer le pouvoir; en revanche, les questions qui touchaient à l'amélioration du quotidien furent, quant à elles, significativement passées sous silence ${ }^{34}$.

Tangible dès les années 1880, la municipalisation de la vie politique - entendue comme une acclimatation réussie de la politique dans le cadre de la communauté d'habitants - supposait ensuite que les aspirants au gouvernement communal sachent faire passer au premier plan tout ce qui relevait de l'idée qu'ils se faisaient de l'intérêt local. L'unanimité autour des aménagements propres à améliorer le sort de la population - ou d'une partie de celle-ci - était subordonnée à une condition que les candidats avaient bien comprise : ne pas apparaître, aux yeux de leurs contemporains dont ils guignaient les suffrages, comme des diviseurs qui n'hésiteraient pas à prendre prétexte pour briser le consensus - avéré ou imaginé - et faire avancer leur cause. L'expérience du socialisme puis du communisme municipal dans les villes portuaires fut à cet égard particulièrement éclairante. Si certaines équipes prirent des décisions marquées au sceau de

32. Damamme, Dominique, "Professionnel de la politique, un métier peu avouable ", dans OfFERLÉ, Michel (dir.), La profession politique, XIXe-XXe siècles, Paris, Belin, 1999, p. 37-67, p. $47-48$.

33. LAGRÉE, Michel, Religion et cultures en Bretagne, 1850-1950, Paris, Fayard, 1992, p. 130132.

34. Le BAIL, GeORGes, Une Élection Législative en 1906 (Miettes électorales). Mémoires précédés d'une étude sur le clergé breton, Paris, Librairie Léon Vanier éditeur, 1908. Décrite a posteriori par le candidat combiste, la campagne électorale lui donna l'occasion de réifier la division entre marins et paysans, cette division qui lui permettait de se présenter comme le défenseur des populations côtières et lui garantissait, ce faisant, de sérieux dividendes électoraux : "Dans un hameau de la commune de Plobannalec nommé Léchouargat, les chouans accourus des communes du canton de Pont-l'Abbé s'étaient réunis dès le matin au nombre de 6 ou 700 pour faire leur affaire au candidat républicain et à ses amis qui devaient y tenir une réunion dans la journée. L'attente prolongée lassa la patience d'un certain nombre d'entre eux qui regagnèrent leur ferme, et, quand je me présentai vers trois heures, escorté de la population voisine de Lesconil, formant une longue colonne de manifestants porteurs de plus de 50 drapeaux et oriflammes, on sentait qu'il y avait de la poudre dans l'air " (p. 186-187). 
l'idéologie qu'ils promurent, aucune, à notre connaissance, ne se risqua à politiser consciemment un projet ressortissant à l'anthropisation volontaire du littoral. L'échec de l'implantation d'un port industriel à Douarnenez, en dépit des nombreuses tentatives qui virent le jour entre 1919 et 1927, ne fut pas tant imputable aux crispations du débat politique liées à l'arrivée des communistes à la mairie qu'aux entraves techniques, économiques (l'absence de soutien de la Chambre de commerce de Quimper) et humaines (l'intransigeance de certains usiniers trop heureux d'utiliser leur pouvoir de nuisance pour perturber les actions de la municipalité révolutionnaire) qui surgirent pour faire capoter le projet ${ }^{35}$.

Enfin, la valorisation du cadre local érigé en socle démocratique par un régime républicain soucieux de fortifier son assise exigeait implicitement des individus siégeant dans les assemblées communales et départementales qu'ils incarnent la vitalité de leur petite patrie. L'élu bâtisseur fut à la fois le produit d'une logique étatique qui dota l'institution municipale d'une capacité d'intervention dans des domaines de plus en plus nombreux - elle l'obligeait, corrélativement, à mettre en place une politique de " grands travaux " - et le bénéficiaire de ladite logique à condition qu'il ait su intégrer dans sa stratégie de conservation du pouvoir un paramètre de plus en plus prégnant : à savoir que sa bonne fortune électorale dépendait tout autant de sa notabilité que des bénéfices qu'il pouvait escompter de son action - réelle et/ou présentée comme telle - à la place (maire, conseiller d'arrondissement, conseiller général) qu'il avait occupée. Pour un élu, l'investissement municipal dans la pierre et la maçonnerie pouvait se révéler être un fructueux investissement électoral à plus ou moins long terme. Une exigence qui supposait, en filigrane, que le mandataire des intérêts de la collectivité sache évaluer au mieux le rapport qui se nouait entre une action généralement coûteuse - les entreprises furent souvent abandonnées faute de ressources communales suffisantes - et les mérites dont ses suffragants le créditeraient pour avoir réussi à mener à bien un tel projet. Ainsi, alors que l'organisation des campagnes électorales incitait de plus en plus les candidats à proposer leur vision du futur ou à se recommander de leur bilan, la valorisation des entreprises en cours ou la promesse des ouvrages à venir participèrent incidemment à la construction de la figure du " bon élu " et de la " bonne assemblée ". Dans sa délibération du 11 février 1906 à propos de la construction d'un avant-port qui devait permettre d'abriter la centaine de bateaux qui fréquentaient les lieux, le conseil municipal de Sauzon rappela : "Quoique la situation financière de la commune n'est pas brillante le conseil fera l'impossible pour payer une part des travaux si le vœu qu'il forme est bien accueilli ${ }^{36}$. " Le soulagement des marins et des pêcheurs n'excluait pas des visées plus politiques.

35. Nous reprenons ici les analyses de Jean-Michel Le Boulanger, Douarnenez de 1800 à nos jours. Essai de géographie historique sur l'identité d'une ville, Rennes, PUR, 2000, p. 239-251.

36. Arch. dép. du Morbihan, 4 S 799. 
Au terme de cette analyse, une constatation s'impose donc : des aménagements portuaires émergèrent dans des programmes politiques locaux au même titre que les autres travaux concernant l'amélioration du quotidien. Pour autant, ils ne furent guère utilisés comme des arguments idéologiques au cours des batailles électorales. L'unanimité prévalut quand bien même elle masquait les ambiguïtés d'un consensus absolu.

\section{Le masque de l'unanimité}

L'unanimité que sécrétait généralement un ouvrage reconnu d'utilité publique subissait pourtant çà et là des lézardes. Les indices sont rares et fragmentaires. Ils attestent toutefois que certains aménagements suscitèrent émois et remous. L'ingénieur ordinaire nota, par exemple, dans son rapport du 24 avril 1890 que les marins du hameau de Rostiviec et le conseil municipal de Loperhet n'étaient pas " entièrement d'accord sur la nature des ouvrages qu'ils demand[ai]ent ${ }^{37}$ ". Conditionné au paiement des trois quarts de la dépense, le projet d'exhaussement de la cale des Salles ne vit finalement pas le jour après que les élus de Riantec eurent décidé, le 3 novembre 1929, "par un vote secret de 16 voix contre $4^{38}$ ", de surseoir aux frais à engager. Ces tiraillements, dont nous pouvons supposer qu'ils étaient liés à des rivalités entre des personnes et à la concurrence d'intérêts privés, ne débouchèrent que très exceptionnellement sur des clivages susceptibles d'affecter la vie municipale. Cas extrême : à Camaret, en 1925, l'élection pour le renouvellement du conseil municipal fut parasitée par la construction d'un gril de carénage. En décidant de ne pas participer à la commission nautique qui devait statuer sur le sort de ce projet dans le seul but de le voir échouer, l'armateur Keraudren, membre correspondant de la Chambre de commerce de Brest et électeur dans la commune voisine de Crozon, avait espéré porter préjudice à son adversaire politique en lui faisant porter l'échec d'un ouvrage attendu par la population depuis longtemps. Dénoncées dans l'affiche que le maire sortant fit placarder avant la tenue du scrutin de ballottage, les manœuvres autour d'un ouvrage portuaire se parèrent, pour une fois, de cette dimension polémique susceptible d'infléchir les résultats de la consultation :

"M. Keraudren a refusé de venir à la réunion de la consultation nautique, malgré l'insistance de tous les présents, car il lui était difficile d'en empêcher la construction après l'avoir demandée. Des ordres étaient donnés, avant, à ses fidèles. Pour la grue, tout le monde est fixé, puisqu'il mâte toujours ses bateaux avec l'ancien système. Il préfère sacrifier son personnel que de se soumettre au progrès des autres. Quant à la profession de foi de sa liste, il est beaucoup plus question de politique que d'intérêts communaux ${ }^{39}$. "

37. Arch. dép. du Finistère, 4 S 1391.

38. Arch. dép. du Morbihan, 4 S 3162.

39. Arch. dép. du Finistère, 3 M 463, affiche de Poupat en vue du scrutin du 10 mai 1925. 
C'est parce que les tensions politiques nées de la volonté d'aménager le rivage furent rares, dans des sociétés locales fréquemment soumises à l'incandescence conflictuelle, qu'il convient de réfléchir en contrepoint à la teneur de cette unanimité communale. Engager l'avenir de la collectivité en faisant d'un brise-lames ou d'un quai ce dénominateur commun à l'ensemble des habitants qui la composaient, c'était, d'une certaine manière, essayer de conjurer les risques de la division fondés sur la singularisation possible des attentes. Dans les circonscriptions littorales qui abritaient des travailleurs exerçant des métiers très différents, la fragmentation du tissu social à partir de la spécialisation grandissante des activités économiques faisait, en effet, planer inévitablement le spectre de la dissociation. Tout aménagement projeté pour répondre aux demandes d'une catégorie de la population était alors susceptible de devenir un point de friction tant il pouvait incarner l'utilisation des finances municipales à des fins particulières. Dans la très vaste commune de Plougastel-Daoulas, le risque de l'éclatement pesa incontestablement sur les choix que durent faire les élus. Presqu'île associant une population de marins-cultivateurs et de paysans domiciliés dans des hameaux qui appartenaient chacun à une frairie, la commune était divisée en micro-territoires aisément identifiables par tous les habitants ${ }^{40}$. Porte-parole de la collectivité, le conseil municipal se devait d'être le représentant de cet enchevêtrement territorial s'il voulait bien fonctionner. Aussi, les demandes concernant la poussière de petits ports installés sur les 36 kilomètres de côte supposaient-elles, afin d'éliminer les tensions sous-jacentes, de suggérer que de tels aménagements constituaient autant d'équipements communs ${ }^{41}$.

Vider tout ouvrage littoral de son contenu potentiellement controversable en ne l'associant pas au seul groupe social à qui il servirait en priorité revenait à continuer d'associer le destin de ce dernier au destin de la communauté dans sa totalité. Si les élus ne formalisèrent pas aussi clairement leur attachement à insérer tout projet dans le cadre d'une politique municipale d'ensemble, l'on notera que leur gestion du dossier matérialisa toutefois ce qui était moins qu'une doxa et bien plus qu'une simple habitude, une règle de conduite en somme : la préservation de l'unité communautaire. C'est en son nom que toutes les hypothèques concourant à son délitement devaient être mises à distance. Revêtus du caractère de biens d'utilité publique, la cale et le quai furent délestés de ce qui en faisait les empreintes d'une identité sociale et territoriale... et donc les marqueurs d'une scission et d'un fractionnement qui risquaient d'être à l'œuvre. L'inscription de l'ouvrage portuaire dans une pensée édilitaire qui privilégiait une définition organiciste du social fut patente

40. Bouchy, Anne-Marie, Plougastel-Daoulas : la vie de la famille et du village. Le cycle de la vie individuelle dans la société paysanne de 1890 à 1940, mémoire de maîtrise en ethnologie (Jean-Marie GuILCHER [dir.]), université de Bretagne Occidentale, 1971, p. 27-33 et 164-174.

41. Arch. dép. du Finistère, 4 S 242 et 4 S 1380. 
à Sibiril. Afin que l'anse de Moguériec pût être dotée au plus vite d'une jetée, le maire, du Rusquec, obéissant à la fois à la tradition de l'évergétisme notabiliaire et à l'idée qu'il se faisait d'un maire garant de l'unité de sa commune, se proposa, en 1894, de prendre à sa charge le coût des travaux évalué à 1400 francs $^{42}$. Dans un contexte différent, le vœu qu'émit le conseil municipal de Plouhinec, le 2 novembre 1891, à propos de l'aménagement du site de Pors Poulhan, trahit une utilisation de l'ouvrage projeté à des fins de resserrement communautaire :

"Les habitants du pays, dont le conseil municipal de Plouhinec s'est fait l'interprète, demandent la construction de jetées pour abriter complètement l'anse contre les vents du Sud. Ils estiment que, grâce à cette construction, l'anse ne tarderait pas à être fréquentée par tous les bateaux de pêche de la région et que de nombreuses usines ne tarderaient pas de venir s'y établir en raison de la proximité des lieux de pêche. En un mot ce serait une transformation complète des conditions commerciales et industrielles de la contrée. Le port d'Audierne qui se trouve plus éloigné des lieux de pêche et dont l'entrée présente quelques difficultés disparaîtrait peu à peu et le port de Poulhan serait destiné à le remplacer ${ }^{43}$."

Le plaidoyer en faveur de la construction des jetées n'obéissait pas seulement à des considérations économiques; promise à un bel avenir dans le cadre de la prospective municipale, la transformation attendue de la petite anse qui accueillait alors une dizaine de bateaux devait favoriser une dynamique de rassemblement populaire. Il ne faisait pas de doute, dans l'esprit des élus, que les profits annoncés à moyenne échéance et l'instrumentalisation du patriotisme de clocher - souder la communauté en proposant qu'elle prospère au détriment de la ville portuaire - sauraient avoir raison des voix qui pourraient s'élever pour protester contre la métamorphose soudaine d'un intérêt sectorisé en une cause commune.

Le caractère apparemment anodin de l'aménagement portuaire ne saurait donc masquer les enjeux sous-jacents d'une myriade d'entreprises qui transformèrent le littoral français. Si la cale ou le quai ne servirent pas d'abcès de fixation idéologique, il n'en reste pas moins que leur construction participa, dans les communes à la fois maritimes et rurales, à la pérennisation d'un idéal, celui de l'unité de la communauté. En acceptant de prendre en compte les revendications de certains de leurs contemporains à condition de les recouvrir de cette unanimité communale, les édiles dépouillèrent, ce faisant, les principaux utilisateurs de ces ouvrages, marins pluriactifs, occasionnels ou navigants permanents, de leurs particularités. Le détournement de sens - du sens minoritaire au sens commun - infligé à l'aménagement du littoral fut à la mesure de l'impossibilité de considérer politiquement la collectivité sous l'angle de sa diversité sociale. Manifestation évidente de l'existence d'une population maritime, la cale et

42. Arch. dép. du Finistère, 4 S 1367.

43. Arch. dép. du Finistère, 4 S 1426, rapport de l'ingénieur ordinaire du $1^{\mathrm{er}}$ août 1892. 
le quai restèrent avant tout les expressions d'une exigence, celle de l'unité communautaire $^{44}$.

\section{RÉSUMÉ}

À la croisée des revendications individuelles et/ou collectives et des attentes de l'État (ou de ses résistances), l'aménagement progressif d'un littoral organisé au service des activités humaines se dote à la fin du $\mathrm{XIX}^{\mathrm{e}}$ siècle de nouveaux enjeux. Alors que le champ politique gagne davantage en autonomie, l'anthropisation volontariste des côtes met en jeu et en ouvre des prises de décision qui relèvent désormais autant d'une compétence et d'un primat techniques et étatiques que d'une implication des élus soucieux d'impulser et de contrôler des projets. L'objet de cet article est de proposer un questionnement sur les modalités de la politisation de l'aménagement du rivage entre 1890 et 1930 .

\section{ABSTRACT}

At the crossroads of individual and/or collective claims and expectations of the state, the gradual development of the coast at the end of the nineteenth century organised to assist human activity resulted in new challenges. While the political field was gaining more autonomy, proactive anthropisation of the coasts involved decisions that now were as much the result of technical and state primacy and competence as of a conscious involvement of elected representatives to initiate and manage projects. The purpose of this paper is to question the terms of the politicisation of shoreline development between 1890 and 1930.

44. L'on pourrait prolonger cette étude en examinant les enjeux que soulevèrent les aménagements littoraux à destination des bateaux de plaisance. L'idéal communautaire nappa-t-il toujours des entreprises qui, avec la progression d'un tourisme littoral, concernèrent davantage les villégiateurs fréquentant les stations balnéaires que les habitants de ces communes côtières? La question reste en suspens. 